\title{
Proper motions in the Galactic bulge: Plaut's window
}

\author{
Katherine Vieira ${ }^{1}$, Dana Cassetti-Dinescu ${ }^{1}$, René A. Méndez ${ }^{2}$, \\ R. Michael Rich ${ }^{3}$, Terrence M. Girard ${ }^{1}$, Vladimir I. Korchagin ${ }^{1}$, \\ William van Altena ${ }^{1}$ Steven R. Majewski ${ }^{4}$ and Sidney van den Bergh ${ }^{5}$ \\ ${ }^{1}$ Astronomy Department, Yale University, P.O. Box 208101, New Haven, CT 06520, USA \\ email: vieira,dana,girard,vik,vanalten@astro.yale.edu \\ ${ }^{2}$ Depto. de Astronomia, Universidad de Chile, Casilla 36-D, Santiago, Chile \\ email:rmendez@das.uchile.cl \\ ${ }^{3}$ University of California, 8979 Math Sciences, Los Angeles, CA 90095-1562, USA \\ email: rmr@astro.ucla.edu \\ ${ }^{4}$ University of Virginia, P.O. Box 400325, Charlottesville, VA 22904-4325, USA \\ email: srm4n@didjeridu.astro.virginia.edu \\ ${ }^{5}$ Herzberg Institute of Astrophysics, 5071 West Saanich Road, Victoria, BC V9E 2E7, Canada \\ email: sidney.vandenBergh@nrc-cnrc.gc.ca
}

\begin{abstract}
A proper motion study of a field of $20^{\prime} \times 20^{\prime}$ inside Plaut's low extinction window $(1, \mathrm{~b})=\left(0^{\circ},-8^{\circ}\right)$, has been completed. Relative proper motions and photographic $B V$ photometry have been derived for $\sim 21,000$ stars reaching to $V \sim 20.5 \mathrm{mag}$, based on the astrometric reduction of 43 photographic plates, spanning over 21 years of epoch difference. Proper motion errors are typically 1 mas $\mathrm{yr}^{-1}$. Cross-referencing with the 2MASS catalog yielded a sample of $\sim 8700$ stars, from which predominantly disk and bulge subsamples were selected photometrically from the $J H$ color-magnitude diagram. The two samples exhibited different proper-motion distributions, with the disk displaying the expected reflex solar motion. Galactic rotation was also detected for stars between $\sim 2$ and $\sim 3 \mathrm{kpc}$ from us. The bulge sample, represented by red giants, has an intrinsic proper motion dispersion of $\left(\sigma_{l}, \sigma_{b}\right)=(3.39,2.91) \pm(0.11,0.09)$ mas yr$^{-1}$, which is in good agreement with previous results. A mean distance of $6.37_{-0.77}^{+0.87} \mathrm{kpc}$ has been estimated for the bulge sample, based on the observed $K$ magnitude of the horizontal branch red clump. The metallicity $[M / H]$ distribution was also obtained for a subsample of 60 bulge giants stars, based on calibrated photometric indices. The observed $[M / H]$ shows a peak value at $[M / H] \sim-0.1$ with an extended metal poor tail and around $30 \%$ of the stars with supersolar metallicity. No change in proper motion dispersion was observed as a function of $[M / H]$. We are currently in the process of obtaining CCD $U B V R I$ photometry for the entire proper-motion sample of $\sim 21,000$ stars.
\end{abstract}

Keywords. astrometry — stars: kinematics — Galaxy: bulge — Galaxy: disk — catalogs

\section{Introduction}

Substantial efforts to probe the structure, dynamics and stellar populations of the bulge of the Milky Way, have been made using data coming from all available wavelengths, nonetheless a clear evolutionary picture for it is still not in hand. In the optical range, low extinction windows provide the only possible opportunity to measure the kinematics of the stars, either through radial velocities or proper motions. All proper motion investigations of the bulge, including the one we present in this paper, more or less agree on a velocity dispersion based on proper motions of around $\sigma_{l}=150 \mathrm{~km} \mathrm{~s}^{-1}$ and 
$\sigma_{b}=110 \mathrm{~km} \mathrm{~s}^{-1}$ in Galactic longitude and latitude respectively. The observed anisotropy $\sigma_{l} / \sigma_{b}$ has been explained by "rotational broadening": bulge rotation produces an apparent increase of $\sigma_{l}$ in an intrinsically isotropic oblate rotator body (Zhao et al. 1996). In this investigation, we work on one of the least studied bulge low extinction fields, the so-called Plaut's window, at $(\mathrm{l}, \mathrm{b})=\left(0^{\circ},-8^{\circ}\right)$. With $E(B-V)=0.25 \pm 0.05 \mathrm{mag}$, (van den Berg \& Herbst 1974), it has a smaller reddening and is less crowded than Baade's window, and probes the interesting transition zone between the metal-rich Galactic bulge and the metal-poor halo.

\section{The Astrometric Reduction}

This investigation is based on 43 photographic plates, taken with five different telescopes (for details, see Vieira et al. 2007). The plates were taken in both $B$ and $V$ passbands, have a baseline of 21 years, and some are deep enough $\left(V_{\text {lim }} \sim 22\right)$ to reach below the main-sequence turnoff of the bulge. The plates were digitized with the Yale PDS 2020G microdensitometer. Details of the object detection and centering precision can be found at Vieira et al. (2007). We obtained $B V$ photographic photometry based on 26 photoelectric standards from van den Bergh \& Herbst (1974). The calibrated $(B-V)$ colors were used in modeling color terms in the astrometric reductions, which are generally due to differential atmospheric color refraction. The proper motions were computed using a central plate overlap method, which provides differential proper motions with respect to a relative reference system. The lack of well measured galaxies made it impossible to provide a reliable zero point for absolute proper motions.

\section{Results}

After cross-referencing our catalog with 2MASS, we used the infrared photometry to cleanly separate bulge from foreground disk stars. Figure 1 (left) shows the $J$ vs. $J-H$ diagram for the Plaut's window. The bulge Red Giant Branch (RGB) can be easily distinguished. The Horizontal Branch (HB) red clump of the bulge can be seen at $J \sim 13.5$ and $J-H \sim 0.6$, with a large magnitude spread due to a combination of differential reddening, metallicity dispersion and depth effect. Just below the HB red clump is the RGB bump, seen at $J \sim 14$ and $J-H \sim 0.6$. Foreground disk main sequence stars are located in the sequence extending upwards $(J \leqslant 16)$ and bluewards $(0.0 \leqslant J-H \leqslant 0.45)$. Another vertical sequence can be distinguished at $J-H \sim 0.6$ for $J<13$, the disk red clump of stars, dispersed in magnitude as a result of their large spread in distance and reddening (Zoccali et al. 2003).

From Figure 1 (left), we selected 482 bulge stars, by using the decontaminated bulge sample of Zoccali et al. (2003) as a template. We also separated a sample of 1851 disk main sequence stars by selecting those with $0.0 \leqslant J-H \leqslant 0.45$ and $J \leqslant 16$. Each population has its own kinematical characteristics, for example, the bulge sample easily stands out from the disk sample, in the $\mu_{l} \cos b$ distribution, while the disk stars exhibit trends in their proper motions consistent with the projection of the reflex solar motion and even Galactic rotation (for stars fainter than $V \sim 16.7$, i.e. distance $\sim 2 \mathrm{kpc}$ ).

The bulge intrinsic proper motion dispersion was calculated from the observed proper motions (Figure 1 right), corrected by the contribution of the estimated errors. We ob-

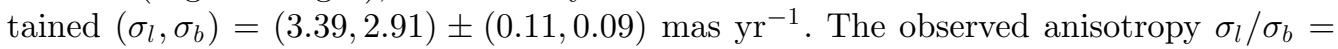
$1.17 \pm 0.05$, can be explained by "rotational broadening", though theoretically speaking, it could also be produced by an intrinsically anisotropic nonrotating bulge. Our result 
compares fairly well with previous studies, but we obtained one of the highest dispersions of all proper motion investigations of the bulge. This can be easily explained by the closeness of our bulge sample. Using the bluge HB red clump $(K \sim 12.67)$ as a distance indicator $\left(M_{K}^{R C}=-1.40 \pm 0.2\right.$, Ferraro et al. 2006), we measured a mean distance to our bulge sample of $\sim 6.37_{-0.77}^{+0.87} \mathrm{kpc}$. This means we are looking $\sim 885 \mathrm{pc}$ south of the Galactic plane. At this position, the model of Zhao (1996) predicts that bulge density is $8 \%$ of the highest central value. Converting the proper motion dispersion into a velocity dispersion, we obtain $\left(\sigma\left(v_{l}\right), \sigma\left(v_{b}\right)\right)=(102.30,87.76) \pm(3.32,2.85) \mathrm{km} \mathrm{s}^{-1}$, which compares well with the prediction by Zhao (1996). We also estimate the rotational velocity at Plaut's location (Zhao et al. 1996, eqns. 1 \& 2), and we obtain $52.57 \pm 8.02 \mathrm{~km} \mathrm{~s}^{-1}$. This value is in agreement with the bulge rotation curve as measured by other authors (Izumiura et al. 1994, Tiede \& Terndrup 1999).

We also studied the kinematics of the bulge blue horizontal branch stars, which comprise a total of 103 stars, located at $-0.5<B-V<0.45$ and $15<V<17$ (see Vieira et al. 2007, Figure 2). Their proper motion distribution looks a bit noisier but very similar to that observed for the bulge RGB stars, which makes a very good case for these stars being bona fide members of the bulge. We obtained $\left(\sigma_{l}, \sigma_{b}\right)=(4.23,3.48) \pm(0.30,0.25)$ mas $\mathrm{yr}^{-1}$, but halo contamination could be responsible for these higher values. Their observed anisotropy is $\sigma_{l} / \sigma_{b}=1.22 \pm 0.12$. This result represents the first kinematic link ever between a metal poor and a metal rich population of the Galactic bulge. Additionally, we studied the metallicity distribution of our bulge sample, by using the "hyperbolas" method of Zoccali et al. (2003), which due to its own limitations, could only be applied on a subsample of 60 stars. A histogram of the metallicities obtained shows a peak at $[M / H] \sim-0.1$ with a somewhat extended metal poor tail, and $\sim 30 \%$ of the stars with $[M / H]>0$, which compares well with previous results (Zoccali et al. 2003). No significant change in the proper motion dispersion is noticed between the metal-poor and the metal-rich stars, but since our sample is small and our abundances are based on photometric indices, we do not take this result as a conclusive one in this regard.

\section{Discussion}

The recent availability of proper motion data in numerous locations on the bulge (Kozlowski et al. 2006, Rattenbury et al. 2007), and the observation of what looks like trends in proper motion dispersion according to $(l, b)$, have prompted the idea that these results may reflect intrinsic kinematic features of the bulge. Previous to these, it was known that proper motion dispersions exhibit trends with metallicity (Spaenhauer, Jones \& Withford 1992, Soto et al. 2006) and distance (Kuijken \& Rich 2002). Regarding metallicity, the observation of a smaller $\sigma_{b}$ for the more metal-rich stars, points to the presence of an intrinsically anisotropic stellar bar. This idea is supported by radial velocity measurements (Rich 1990, Minniti 1996) and by the significative vertex deviation observed by Soto et al. 2006 for metal rich stars. Nonetheless, other authors (Tiede \& Terndrup, 1997) attribute these correlations to contamination by disk giants. As for distance, Kuijken \& Rich (2002) were able to probe proper motion dispersion along their line of sight using HST data. They found that the observed proper motion anisotropy was largely caused by the line-of-sight gradient of the rotation of the bulge. Once this is removed, the bulge exhibits a nearly isotropic velocity distribution. This rotation, always assumed to be of a solid body, may actually have some gradient along $b$ (compare rotation rates of Izumiura et al. 1994 and Tiede \& Terndrup 1999 with Rich et al. 2007), and also flattens out for $|l|>5$ (Rich et al. 2007). 

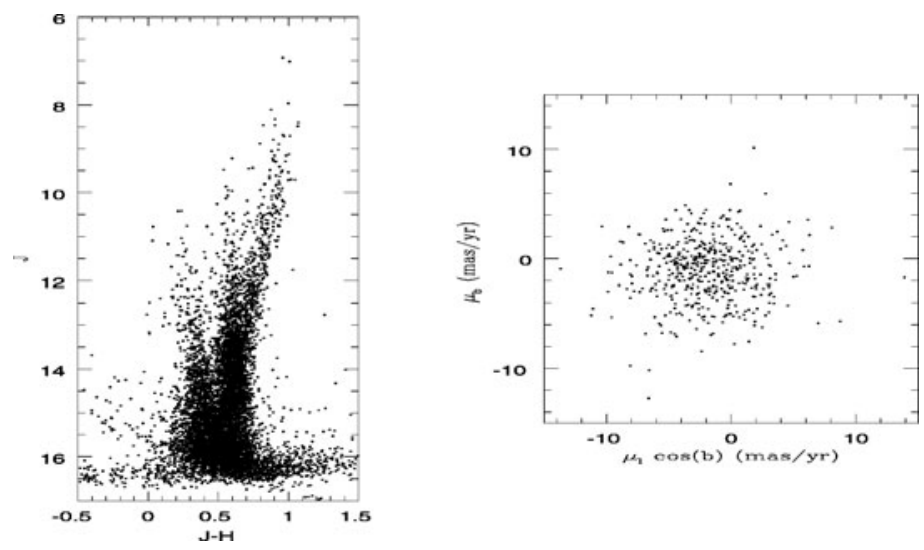

Figure 1. Left: Infrared CMD for the stars in Plaut's window. Disk and Bulge samples are seen in black. Right: Vector-Point Diagram for the bulge sample.

As for variations in velocity disperson according to $(l, b)$, Rich et al. (2007) has found an increase in radial velocity dispersion for those fields closer to the galactic plane. Similarly Kozlowski et al. (2006) and Rattenbury et al. (2007) found increased values of proper motion dispersion for lower $|b|$ and $|l|$ as well. Nevertheless, these two last results fail to agree with each other in the trends they observe. Moreover, in both cases, the selection of their samples is significantly affected by disk contamination, either from distant stars closer to the galactic center, for the case of Kozlowski et al. (2006), or from more nearby disk giants for Rattenbury et al. (2007). Both cases are extensively discussed in Vieira et al. (2007), where we explain how nearby intrinsically faint disk stars will broaden the observed $\sigma_{l}$ over large fields of view, while when observing deep into the galactic center over a very small field of view, many distant disk stars could decrease $\sigma_{l}$. In any case, whether the above observed trends of proper motion dispersion with position are real or not, it is time for the theoretical models to start exploring this subject in more detail, as to offer an insight into this very interesting topic.

\section{References}

Ferraro, F. R., Valenti, E. \& Origlia, L. 2006, AJ, 649, 243

Izumiura, H. et al. 1994, ApJ, 437, 419

Kozlowski, S. et al. 2006, MNRAS, 370, 435

Kuijken, K. \& Rich, R. M. 2002, AJ, 124, 2054

Minniti, D. 1996, ApJ, 459, 579

Rattenbury, N. J. et al. 2007, arXiv:0704.1619 v1

Rich, R. M. 1990, ApJ, 362, 604

Rich, R. M. et al. 2007, AJ, 658, L29

Soto, M., Rich, R. M. \& Kuijken, K. 2006, ApJ, 665, L31

Tiede, G. P. \& Terndrup, D. M. 1997, AJ, 113, 321

Tiede, G. P. \& Terndrup, D. M. 1999, AJ, 118, 895

Spaenhauer, A., Jones, B. F. \& Whitford, A. E. 1992, AJ, 103, 297

van den Bergh, S. \& Herbst, E. 1974, AJ, 79, 603

Vieira, K. et al. 2007, AJ, 134, 1432

Zhao, H. 1996, MNRAS, 283, 149

Zhao, H., Rich R. M. \& Biello, J. 1996, AJ, 470, 506

Zoccali, M. et al., 2003, A\&A, 399, 931 